\title{
Hydrodynamic fingering instability of driven wetting films: hindrance by diffusion
}

\author{
P Carles, S M Troian†, A M Cazabat and F Heslot \\ Collège de France, Physique de la Matière Condensée, 11 place Marcelin Berthelot, 75231 \\ Paris Cédex 05, France
}

Received 30 July 1990

\begin{abstract}
Recent experimental and theoretical efforts have revealed the existence of a fingering instability at the moving front of thin liquid films forced to spread under gravitational, rotational or surface shear stresses, as for example by using the Marangoni effect. We describe how the presence of a precursor film in front of the spreading macroscopic film, whether it is by prewetting the substrate or by surface diffusion or multilayer absorption, can prevent the development of the instability.
\end{abstract}

\section{Introduction}

Thin films of liquids can be made to rise above the equilibrium meniscus position under the force of an upwardly directed surface tension gradient as first studied by Ludviksson and Lightfoot [1]. Such 'super-meniscus' films are formed by using the Marangoni effect [2] to create a surface shear stress on the thin film to force it to spread upwards along the solid surface. Marangoni forces are created by applying a thermal gradient along a vertical surface, which gives rise to a surface tension gradient in the film along the direction of flow: $\tau=\mathrm{d} \gamma / \mathrm{d} x=(\mathrm{d} \gamma / \mathrm{d} T)(\mathrm{d} T / \mathrm{d} x)$, where $\gamma$ is the surface tension, $T$ the temperature and $x$ the distance measured upwards along the plate (figure 1). The variation $(1 / \gamma)(\mathrm{d} \gamma / \mathrm{d} T)$ in surface tension is fairly constant for many fluids far from the critical point, and, for a constant temperature gradient, one can therefore create a constant Marangoni surface stress $\tau=\mathrm{d} \gamma / \mathrm{d} x$.

\section{Experimental and theoretical findings}

As previously reported [3], we have found that the spreading of such films can give rise to a fingering instability at the moving front. The instability is directly connected to the fact that the advancing film front thickens with time and develops a steep 'bump' which goes unstable and breaks into fingers in a manner not unlike the Rayleigh instability [4] (which causes a column of fluid to break into droplets), although the detailed flow is more complex. Since the experimental and theoretical details of our work appear elsewhere $[3,5,6]$, we here briefly describe our set-up and findings. What we wish to

$\dagger$ Permanent address: Exxon Research \& Eng. Co., Clinton Twp., Route 22E, Annandale, NJ 08801, USA. 


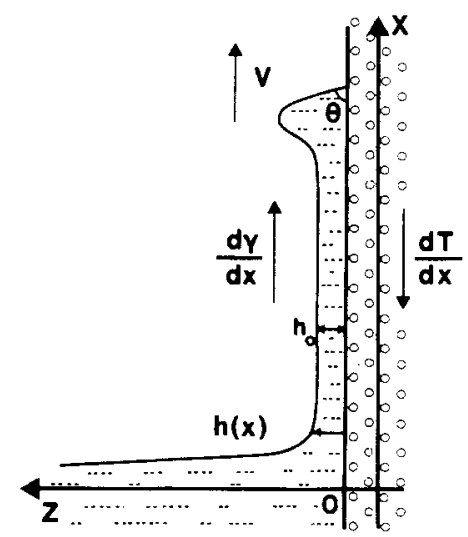

Figure 1. A schematic view of the capillary rise geometry in which a film of PDMS is made to climb under a gradient in surface tension. The macroscopic dynamic contact angle, measured from the reconstructed profiles and listed in table 1 , is denoted by $\theta$.

emphasize in this paper is that the instability disappears with the disappearance of the 'bump' in the height profile. In particular, in comparing our results with the previous work [1], we find that, while surface diffusion is generally not important in our experiments in which the flow is purely hydrodynamic in nature, diffusion did play a significant role in [1], contributing to the disappearance of the 'bump' and therefore the absence of the fingering instability.

In the lubrication approximation, the Navier-Stokes equations describing such unidirectional flow as in figure 1 reduce to

$$
\eta \partial^{2} v / \partial z^{2}=\partial p / \partial x+\rho g
$$

where $v(x, z)$ is the fluid velocity upwards along the plate, $z$ measures the distance from the solid, $\eta$ is the viscosity, $p$ is the pressure in the fluid and $\rho g$ the gravitational force per unit volume. For small surface slopes, $\partial p / \partial x=-\gamma \partial^{3} h / \partial x^{3}$, where $h(x)$ defines the film surface. Integrating equation (1) subject to the boundary conditions $v(x, z=0)=$ 0 and $\eta(\partial v / \partial z)[z=h(x)]=\tau$ gives the height-averaged velocity as

$$
V(x)=(h / 2 \eta) \tau+(\gamma / 3 \eta) h^{2}\left(\partial^{3} h / \partial x^{3}\right) .
$$

The first term describes the climbing of the film under Marangoni forces while the second term describes flow induced by surface curvature. (We only consider thin films where $\rho g h \ll \tau$.) Away from the sharply curved ends of the meniscus region near the bottom of the plate and near the advancing front, surface curvature is negligible and the fluid velocity is dominated by the first term. Under quasistatic conditions in which the incoming flux $Q=h V$ is constant, $h(x)$ will be nearly flat and the fluid film should spread linearly in time with a constant velocity $V_{\text {th }}$ given by

$$
V_{\text {th }}=h_{0} \tau / 2 \eta
$$

where $h_{0}$ is the thickness of the flat film. Near the advancing front where the fluid surface curves sharply to meet the substrate both terms balance, and the length $l$ over which the capillary force is comparable with the viscous and Marangoni forces is

$$
l=h_{0}(3 \mathrm{Ca})^{-1 / 3}
$$

where the capillary number is $\mathrm{Ca}=\eta V_{\mathrm{th}} / \gamma$. As first suggested by Huppert [7] and recently calculated explicitly [6], the length of this capillary region is what determines the scale for the wavelength $\Lambda$ of the fingering instability. 


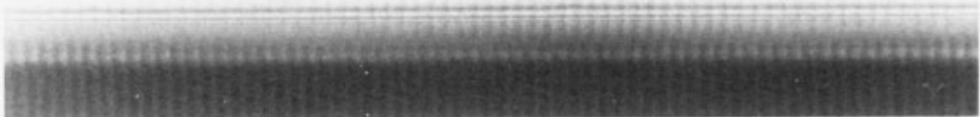

(a)

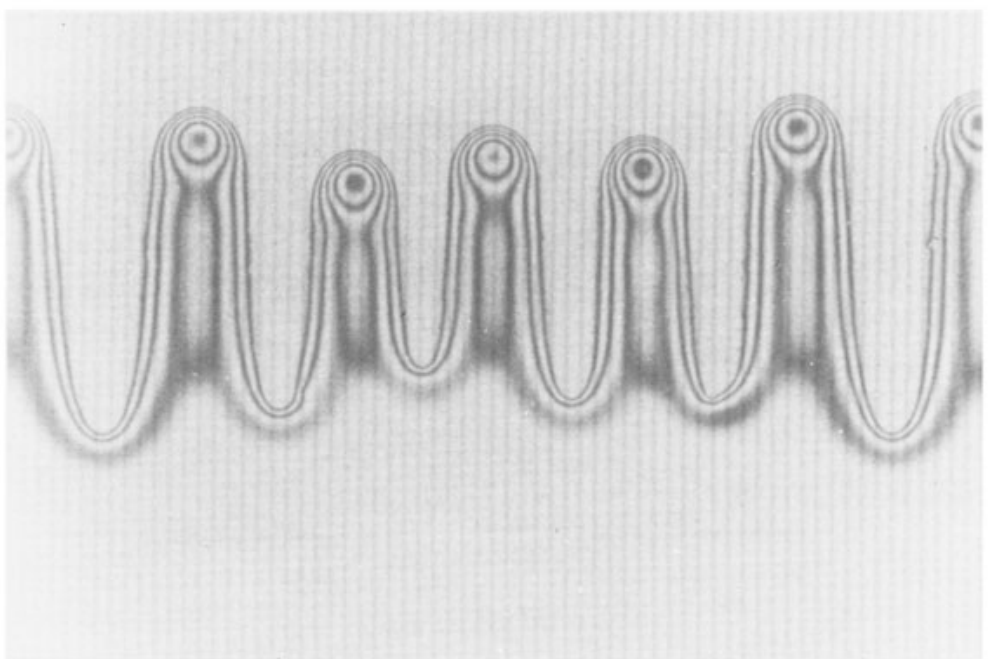

Figure 2. Time development $(a)$ after $5 \mathrm{~min}$ and $(b)$ after $25 \mathrm{~min}$ of the fingering instability at the vertically ascending front of a PDMS oil film of viscosity $20 \mathrm{mPa}$ and $\tau=0.18 \mathrm{~Pa}$ observed under laser illumination. Measured from finger tip to tip, the wavelength $\Lambda$ in $(b)$ is $0.48 \mathrm{~mm}$.

The climbing film is observed through a reflection microscope with laser illumination $(\lambda=6328 \AA$ ). Equal-thickness fringes, spaced $\lambda / 2 n$ apart (where $n=1.4$ is the optical index of the PDMS oil that we used in all our experiments), are used to reconstruct the thickness profiles. Some time after the liquid is put into contact with the vertical silicon wafer, a film of relatively constant thickness begins to climb the wafer. A bump in the height profile, as indicated by the closely spaced fringes, develops near the climbing front (figure $2(a)$ ), which soon becomes unstable and results in a fingering instability with a rather well defined wavelength (figure $2(b)$ ). Figure 3 shows the position of the fluid front as a function of time. The fork in the curve signals the beginning of the 


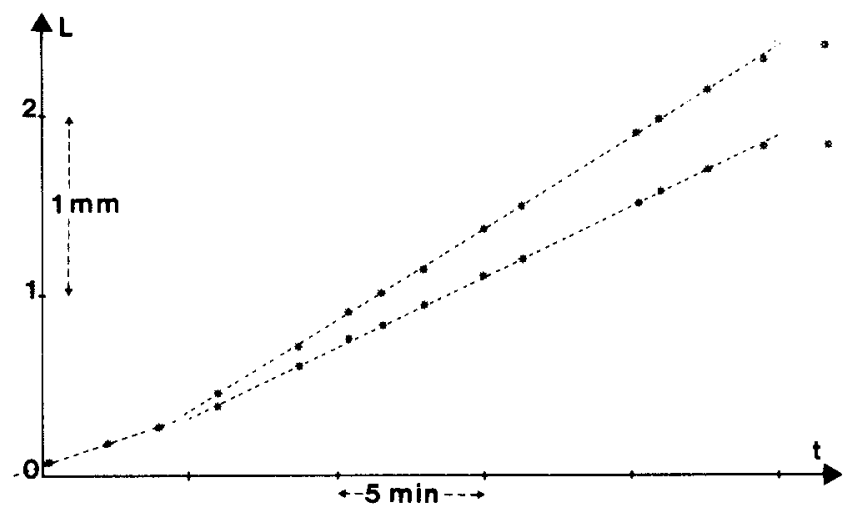

Figure 3. Position of the fluid front as a function of time for PDMS of viscosity $20 \mathrm{mPa}$ and $\tau=0.10 \mathrm{~Pa}$. The values $V$ in table 1 are determined from the slope of the linear regime occurring before the onset of the instability.

instability, the upper curve corresponding to the finger tips and the bottom curve to the 'valleys'.

The height profiles in the absence and presence of the instability as well as the growth rate of the instability as a function of wavenumber have been calculated in a linear stability analysis [6]. This analysis, which gives an estimate of the wavelength $\Lambda$ which is in very good agreement with our experiments, shows that the pronounced bump observed in the height profile causes a capillary-driven fingering instability more complex and yet not unlike the Rayleigh instability.

\section{Comparison with the experiments in [1]}

Although the experimental set-up in [1] is the same as ours, we would like to discuss one possible reason for which the instability did not occur in the previous work. In particular, we here describe processes occurring at the front of the bulk film in [1] which can preclude the development of the instability we found. Our observations lend further weight to the conclusion that the fingering process can only occur in the presence of well developed 'bump' in the height profile.

In the experiments in [1] (see table 1), the Marangoni stress created by the temperature gradient was in general an order of magnitude smaller than the stresses present in our case and so the thin films were not being pulled up the plate as forcefully. The spreading velocities were therefore much smaller than the velocities we observed. This decrease in film velocity, coupled with the fact that the substrates in [1] were prepared by first prewetting them with the oil film, considerably changed the dynamics near the moving contact line. In particular, while we have shown our spreading process and the ensuing instability to be completely hydrodynamic in nature, the experiments in [1] introduced the possibility of multilayer absorption and/or diffusion at the moving edge.

One test of whether the spreading of the front is governed solely by hydrodynamics is to compare the measured contact angles with those calculated from Tanner's [8] relation, which states that for small contact angles the contact angle scales with the capillary number according to $\theta \simeq 3.6(\mathrm{Ca})^{1 / 3}[9]$ (with $V$ the film velocity in the absence 
Table 1. Results for the spreading of PDMS $\left(\eta=0.02 \mathrm{~Pa} \mathrm{~s}\right.$ and $\left.\gamma=0.02 \mathrm{~N} \mathrm{~m}^{-1}\right)$ on a silicon wafer and squalane $\left(\eta=0.028 \mathrm{~Pa}\right.$ s and $\gamma=0.028 \mathrm{~N} \mathrm{~m}^{-1}$ ) on a silver-coated wafer, for our experiments and for those of Ludviksson and Lightfoot, labelled [3] and [1] respectively. Both liquids wet their substrates completely. The asterisks $\left({ }^{*}\right)$ indicate the measurements for which the linear regime was too short to obtain a precise reading. The values of the contact angles $\theta$ for [3] are lower bound estimates since the fringes, being very closely spaced near the moving front, make it difficult to obtain very accurate readings.

\begin{tabular}{llllllll}
\hline Reference & $\begin{array}{l}\tau \\
(\mathrm{Pa})\end{array}$ & $\begin{array}{l}h_{\|} \\
(\mu \mathrm{m})\end{array}$ & $\begin{array}{l}V \\
\left(\mu \mathrm{m} \mathrm{s}^{-1}\right)\end{array}$ & $\begin{array}{l}\theta_{\text {(anner }} \\
(\mathrm{deg})\end{array}$ & $\begin{array}{l}\theta \\
(\mathrm{deg})\end{array}$ & $\begin{array}{l}V_{\mathrm{D}} / V, \\
5 \mathrm{~min}\end{array}$ & $\begin{array}{l}V_{\mathrm{D}} / V \\
60 \mathrm{~min}\end{array}$ \\
\hline$[3]$ & 0.5 & 0.86 & $8^{*}$ & 0.07 & 0.05 & 0.06 & 0.017 \\
{$[3]$} & 0.27 & 0.65 & $3^{*}$ & 0.05 & 0.05 & 0.15 & 0.05 \\
{$[3]$} & 0.21 & 0.54 & 3 & 0.05 & 0.04 & 0.15 & 0.05 \\
{$[3]$} & 0.10 & 0.27 & 1 & 0.036 & 0.03 & 0.5 & 0.13 \\
{$[3]$} & 0.054 & 0.17 & 0.3 & 0.024 & - & 1.5 & 0.5 \\
{$[1]$} & 0.018 & 2.35 & 0.33 & 0.025 & 0.006 & 1.0 & 0.3 \\
{$[1]$} & 0.013 & 1.7 & 0.15 & 0.019 & 0.0035 & 2.2 & 0.6 \\
{$[1]$} & 0.009 & 1.1 & 0.06 & 0.014 & 0.00075 & 5.5 & 1.6 \\
\hline
\end{tabular}

of the instability). In table 1 we show that for our experiments the measured values of the contact angles $\theta$ agree well with the values $\theta_{\text {Tanner }}$ calculated from the Tanner relation. We extracted the analogous quantities from figure 10 and table 1 of [1] and it is clear that Tanner's relation does not hold in their experiments. The measured contact angles are much smaller than those expected just from hydrodynamic considerations, implying that some sort of diffusive or absorptive process occurs at the moving contact line, as Ludviksson and Lightfoot [1] originally suspected.

We expect that such processes lead to the development of a primary or precursor film in the experiments in [1]. In fact, we can calculate the importance of such a diffusive film ahead of the macroscopic front by the following argument. The characteristics of thin precursor films spreading ahead of a macroscopic film of squalane or PDMS oils are known. The length of such precursor films grows as $\sqrt{D t}[10]$, where $D$ is a diffusion constant and $t$ the time, and the growth velocity of such a film is therefore $V_{\mathrm{D}} \simeq$ $0.5 \sqrt{D / t}$. (The diffusion constant for squalane is $1.3 \times 10^{-10} \mathrm{~m}^{2} \mathrm{~s}^{-1}$ and for PDMS $2.8 \times 10^{-10} \mathrm{~m}^{2} \mathrm{~s}^{-1}[11]$.) In table 1 we have calculated the ratio of $V_{\mathrm{D}} / V$ to indicate the relative importance of a diffusive precursor film. Except for the smallest surface tension gradient that we created, diffusion is not an important process for our films. On the other hand, the velocity due to diffusion is comparable with or very much larger than the Marangoni velocities created in [1], implying that a primary film forms ahead of the macroscopic front.

\section{Conclusion}

One reason for which the fingering instability that we are studying was never seen in [1] may be related to the precursor film. The instability, as we understand it, can only form in the presence of a 'bump' in the height profile. With the presence of a precursor film, as in [1], fluid can flow more easily from the macroscopic film into the precursor layer and it is as if a channel for flow exists at the front. This could eliminate a thickening of the fluid at the front and along with it the fingering instability. We conclude that such 
instabilities as we have described could possibly be eliminated by decreasing the front velocity sufficiently that diffusion or absorption processes can play a role or likewise by forcing the film to spread on an already wetted surface. We are also investigating the possibility of the height profile showing no bump either for cases in which $\rho g h$ is comparable to $\tau$ (i.e. thick films).

\section{References}

[1] Ludviksson $V$ and Lightfoot E N 1971 AIChEJ. 17 1166-73

(2) Levich V G and Krylov V S 1969 Ann. Rev. Fluid Mech. 1293

[3] Cazabat A M, Heslot F, Troian S M and Carles P 1990 Nature 346824

[4] Rayleigh Lord 1892 Phil. Mag. 34145

[5] Troian S M, Herbolzheimer E. Safran S A and Joanny J F 1989 Europhys. Lett. 10 25-30

[6] Herbolzheimer E and Troian S M 1990 to be published

[7] Huppert HE 1982 Nature 300 427-9

[8] Tanner L H 1979 J. Phys. D: Appl. Phys. 121478

[9] Hoffman R L 1983 J. Colloid Interface Sci. 94470

[10] Joanny J F and de Gennes P G 1986 J. Physique 47121

[11] Cazabat A M, Fraysse N and Heslot F 1990 Colloids Surf. at press 